\title{
Human-Robot Cooperation through Brain-Computer Interaction and Emulated Haptic Supports
}

\author{
Marie-Pierre Pacaux-Lemoine, Lydia Habib \\ Univ. Valenciennes, CNRS UMR 8201- LAMIH, F-59313 \\ Valenciennes, France \\ Email: \{marie-pierre.lemoine; lydia.habib\}@univ- \\ valenciennes.fr
}

\author{
Tom Carlson \\ University College London, \\ RNOH, Stanmore, HA7 4LP, UK \\ Email: t.carlson@ucl.ac.uk
}

\begin{abstract}
The study presented in this paper is in the context of providing a telepresence platform for people with tetraplegia, who may be confined to their room or bed. The eventual aim is to provide these people with a system that allows them to remotely control a robot, which can act as a medium for him/her in their everyday life, e.g. by enabling interactions with friends and relatives who may be located in other rooms or even remote places and exploring different environments. In this paper, we deal with the specific challenge of cooperation between a robot and a human, who is only able to control the device using thoughts alone. The system is therefore composed of a brain computer interface $(B C I)$ and a visual interface to implement an "emulated haptic shared control" of the robot. The aim is to share motion control between the human and the robot according to the difficulty of the situation. The control schema that exploits this "emulated haptic feedback" has been designed and evaluated using human-machine cooperation (HMC) and has been compared with more standard controls. We report on an initial experiment that has been conducted to test the feasibility of the approach. Preliminary results highlight the interest of the approach but also the challenges that remain to be overcome.
\end{abstract}

Keywords-disability; brain-computer interface; humanmachine cooperation; adaptive level of automation

\section{INTRODUCTION}

Our ultimate aim is to improve quality of life for people with severe tetraplegia, who are perhaps confined to bed and are unable to use conventional interfaces. We intend to empower them to interact with their relatives, friends and environment through the use of a brain-controlled telepresence system. In this work, we are specifically concerned with how the human will be able to operate the device effective, so we apply the principles of human-machine cooperation (HMC) to design such a system.

The principles of HMC have been used in many different domains [1][2] and especially in robotics [3]. The main purpose is to propose a generic approach that helps and assists designers in identifying how the human interacts with a machine, and in implementing the suitable adaptive levels of automation [5], whilst guaranteeing their performance, safety and security [4].

In this paper, we first discuss key concepts in the fields of $\mathrm{HMC}$ and brain-computer interfaces, before considering the specific challenges of our cooperative system. We then present our implementation and feasibility tests, before discussing the results, considering metrics of both task performance and human factors. Finally we conclude that the approach is feasible, but needs further refinement and, in particular, in-depth user acceptance testing.

\section{RELATED WORK}

In this section we review the state-of-the-art and discuss the key concepts in: cooperative agent models; brain-computer interfaces (BCI); and cooperative design.

\section{A. Cooperative agent model}

Human-machine cooperation studies lead to define a model of a cooperative agent with two main dimensions: the agent's ability to control the process, called the know-how (KH); and the agent's ability to cooperate with other agents involved in the process control, called the know-how-to-cooperate (KHC) (cf. Fig. 1).

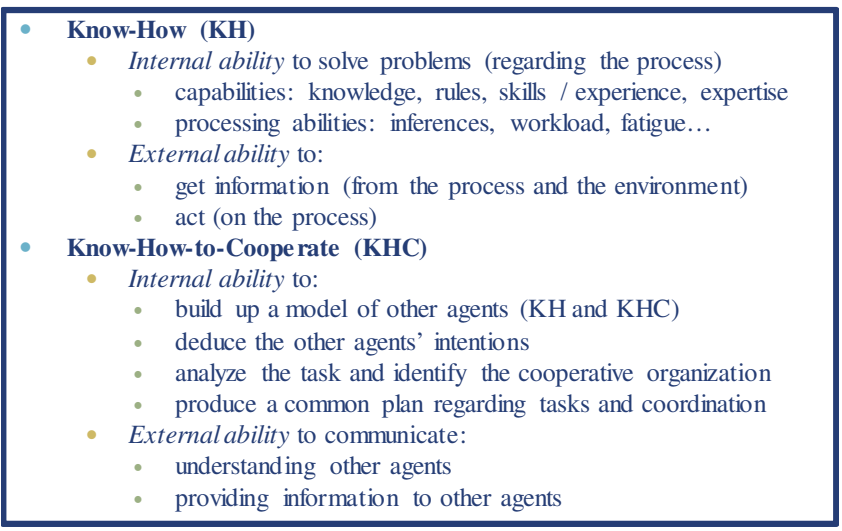

Fig. 1. The model of cooperative agent that we use [8].

The Know-How of an agent only concerns the control of the process, i.e. the achievement of individual tasks without taking into account potential interaction with other agents. The $\mathrm{KH}$ is split up into two parts, one is called the internal $\mathrm{KH}$, the other the external $\mathrm{KH}$. The internal $\mathrm{KH}$ relates agents' competences (abilities) and capacity (workload or attention for example) to control the process. The competence of a human agent is mainly composed of knowledge, rules and skills to control the process [6]. It is linked to expertise, experience and practices of agents. Internal $\mathrm{KH}$ deals with the analysis of information getting from the process in order to identify its state (diagnosis/prognostic) and to make decision regarding its control. Whereas external KH deals with information gathering and action execution. It deals with the ability to get information from the process and the ability to act on the process. It relates ergonomics aspects, regarding data visibility, readability and comprehensibility. Agents have to be aware of their abilities to have information from the process and to act on the process in order to feel in control of the situation. 
To sum up, the internal $\mathrm{KH}$ allows agents to build up a representation of the current and future situation of the process using their competences and capacities to analyze the situation and to make decisions. Agents are able to conduct those functions because they have an interaction with the process through their external $\mathrm{KH}$, to gather information and to implement actions. But other agents take part in the process as well and interact with others with their KHC.

The Know-How-to-Cooperate is also split into two parts. The external KHC is the ability of an agent to have information about other agents and to provide information to other agents. Three main ways are identified in order to reach those goals: they make direct observations of others (movements, mimics, emotions...); they have verbal exchanges or communicate with mediated supports; they analyze the activity of others through the effect of their actions on the process. The support of the external KHC is called the Common Work Space [7]. It supports the situation awareness dedicated to process state and environment, but it is enriched by the team situation awareness dealing with past, current and future activity of other agents [8]. The internal KHC allows agents to build up a model of others in order to make cooperation with them easier. It is built up and updated by learning, training and exchanging with other agents. Agents gather and analyze information about others in order to infer their $\mathrm{KH}$ and $\mathrm{KHC}$.

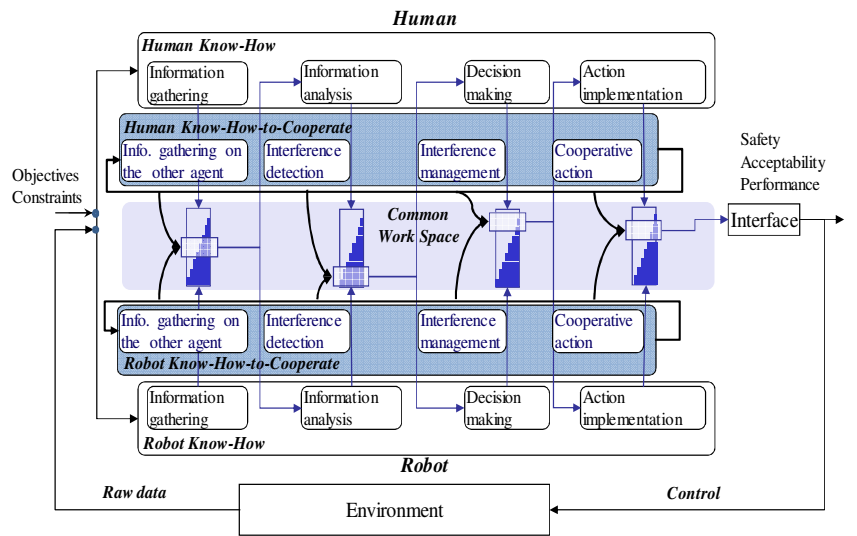

Fig. 2. The model of cooperative activities that we use [9].

$\mathrm{KH}$ and $\mathrm{KHC}$ can be considered as two parallel functions, each one described by four sub-functions. Fig. 2 uses the cooperative agent model to highlight interaction between a human and a machine. KH functions of agents interact within the Common Work Space, represented by the blue rectangle. KHC functions of agents use information provided by the Common Work Space in order to build up a model of the other agent and to evaluate this agent's involvement in the process control. The results of the evaluation compared to their own involvement and model of oneself (interference detection and management) have to lead to the adjustment of the position of the four sliders that describe the functions allocation (scales represented in the Common Work Space). The scales associated with each KH function, (Fig. 2) have the same objectives as the scale proposed by Flemisch et al. [11]. The position of the slider on the scale defines the degree or the percentage of sharing between human and machine activity. Some functions can be completely allocated to machine or to human regarding their competency and capacity, but also regarding how they can take into account the activity of the other. The function allocation can be predefined or updated according to the information on the current situation.

The model of cooperative agents' interactions is useful to analyze the interaction between human and machine inside one level of activity, but it would be also useful to analyze interactions between levels of activity. Goals, decisions and orders evolve from high decisional levels to lower levels closer to the command/control of process. By contrast, events and information update stem from lower levels and are propagated to upper levels. These levels of activity, also called layers [12], are most of the time described as operational, tactical and strategic levels. Machines at tactical and strategic levels are usually decision assistance systems, but at the operational level machines can act on the process and the environment.

All these definitions and concepts have been used to identify and design cooperation between a robot and a human through the Brain-Computer Interface system that is now presented.

\section{B. Brain-Computer Interface (BCI)}

Our Brain-Computer Interface (BCI) is built upon the Motor Imagery platform developed by CNBI, EPFL [13]. In this paradigm, we are able to detect when the user is imagining moving either their left or right hand, or neither, which yields two explicit classes that can be used for control. When the user does not imagine moving either hand, a third implicit control class exists, whereby the control authority is passed to the robot itself. This type of BCI has been demonstrated to be a potentially viable control input in many different application areas, ranging from wheelchairs [14] to exoskeletons [15].

Taking the same approach as EPFL, we record the electrical activity of the brain, in a non-invasive manner, using a process called electroencephalogram (EEG). To achieve this, we placed 16 electrodes (g.GAMMA by g.Tec $\mathrm{GmbH}$ ) on the scalp, over the Motor Cortex and connect them to a laptop via the g.USBamp biosignal amplifier. The EEG was recorded at $512 \mathrm{~Hz}$ and Laplacian filtered to improve the signal-to-noise ratio. The power spectral density (PSD) was then estimated over the last second, in the $4-48 \mathrm{~Hz}$ band with a $2 \mathrm{~Hz}$ resolution [16]. These PSD features were calculated every $62.5 \mathrm{~ms}$ (i.e. 16 times per second) using the Welch method with 5 overlapped (25\%) Hanning windows of $500 \mathrm{~ms}$. We were then able to select the features that best reflect the motor-imagery task for each individual participant using canonical variate analysis (CVA), which maximizes the separability between the different tasks and that are most stable [17]. We then trained a Gaussian classifier using these features, which typically fell in the muband $(\sim 8-13 \mathrm{~Hz})$, in line with the motor control literature. Finally, to improve the individual command accuracy and prevent accidentally command delivery, the instantaneous classifier outputs were accumulated using an exponential smoothing probability integration framework [18]. Once this value surpassed a subject-specific threshold, a command was delivered; e.g. if left hand imagery was detected, a turn left command was issued. 


\section{HMC vs Human-BCI-robot cooperation design}

The human-machine cooperation approach has been used to identify human and machines abilities regarding their individual and cooperative tasks. The $\mathrm{KH}$ and the $\mathrm{KHC}$ have been defined for the human and for the combined entity of the robot and the BCI. We consider that $\mathrm{KH}$ and $\mathrm{KHC}$ are two parallel functions, each one identified by four sub-functions. Moreover, $\mathrm{KH}$ and $\mathrm{KHC}$ are different according to the level of activity on which they are used. This study implements two levels: the tactical and the operational level. The operational level focuses on the obstacle avoidance. The tactical level manages the update of the mission plan: i.e. how to modify the trajectory to reach the target, after the avoidance of an unexpected obstacle.

In this study dealing with telepresence, we consider that the target does not impose the design of a precise plan, because it could be changed as and when different opportunities arise for the human (other information to search, other people to meet...). Moreover, it could be difficult for the human to provide precise details of the target to the robot, due to the relatively low resolution and throughput of the BCI as an interface. In this case, the robot is not able to know the target explicitly and to modify the trajectory itself to reach it. Therefore the robot/BCI system has so no $\mathrm{KH}$ and $\mathrm{KHC}$ at the tactical level.

On the other hand, at the operational level, both human and robot have the abilities to control obstacle avoidance. The human can detect obstacles thanks to a live video stream of the environment from the robot's perspective; and the robot can detect obstacle thanks to its actuated ultrasonic sensor. Both can make decision but only the robot can apply the decision by modifying its trajectory (cf. Fig. 3: Know-How).

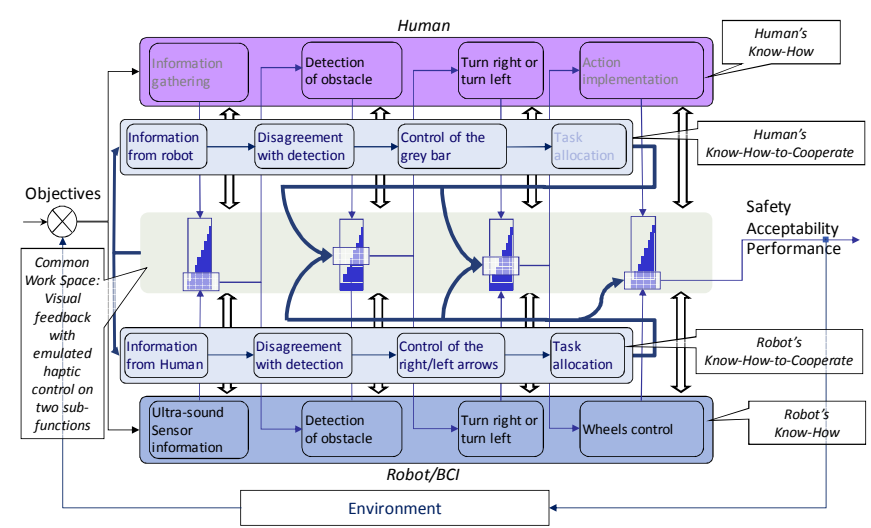

Fig. 3. Use of the cooperative agent model to specify Human-BCI-robot cooperative system. Some sub-functions are not available for Human. Therefore, shared control is on the obstacle detection and avoidance command

Both human and robot have information about each other. They detect and manage interference about obstacle detection and command through the Common Work Space (cf. Fig. 3: Know-How-to-Cooperate). But the last decision concerning command (right or left) is made by the robot, due to the human's limited capacity to react quickly to a situation (which is an inherent constraint of the current state-of-the art in this type of BCI).
The "emulated shared control" is based on the control of interference detection and management of the KHC. This new concept has been defined with the same idea as the haptic control used in robotics and aeronautics (force feedback in the joystick) and in car driving (force feedback in the steering) [19]. In the case of a command sent via a BCI, there is no real haptic feedback because no muscles are involved in the control to oppose or follow the direction provided by the system. But the idea is to emulate this haptic behavior with a visual display. Depending on where obstacles are detected, the robot/BCI system makes it easier or more difficult-in terms of (mental) effort-for the human to deliver a command (cf. Fig. 4).

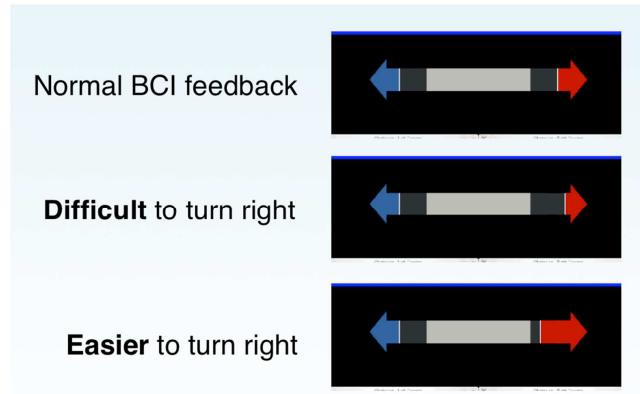

Fig. 4. The user moves the grey bar to the left and right by performing motor imagery. When the bar hits one of the arrows, it delivers the corresponding turn left or right command. Dynamically changing the thresholds to deliver BCI commands is illustrated by changing the size of the arrow and essentially makes it (mentally) easier or more difficult to turn in one or both directions. This gives the illusion of a "haptic" feedback, although no real contact forces or muscle activation are involved.

This new concept has been implemented and evaluated with experiments now presented.

\section{IMPLEMENTATION AND TESTS}

The experiment is a pilot study that aims to verify the technical feasibility and human acceptance. The complete experimental protocol is explained in the following parts.

\section{A. Use case study}

Our use case is: "The user (human) wants to move the robot in a room in order to see something or meet someone and has to come back to the start point."

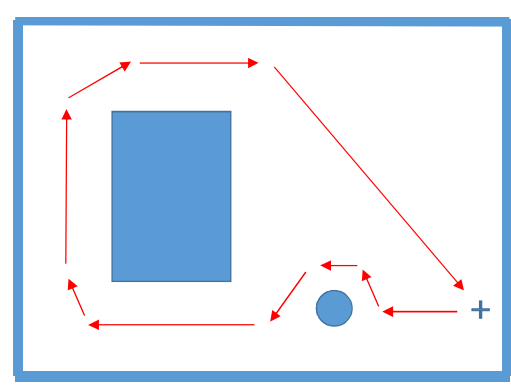

Fig. 5. Experiment room: the goal of the mission is to control the robot in order to follow a trajectory similar to the red track.

The experimental room consisted of two obstacles. The optimal trajectory should be the red trajectory (cf. Fig. 5). 


\section{B. Experimental platform}

\section{1) Mobile robots}

The mobile robot is built using Lego Mindstorms NXT (cf. Fig. 6). It is a low cost and useful small robot, enabling rapid prototyping of human-robot interactions. The embedded computational capabilities are provided by a 32-Bit ARM CPU clocked at $48 \mathrm{Mhz}$ assisted by a co-processor ATmega clocked at $8 \mathrm{Mhz}$. It has 3 sensors (ultrasonic, gyroscope and touch) and a fourth input is used for XBee communication. It is equipped with 3 motors: two for wheels and one to actuate the ulrasonic sensor in a planar, lateral sweeping motion. The robot is programmed in order to avoid collisions with obstacles, but the user can provide new sub-goal directions at any point.

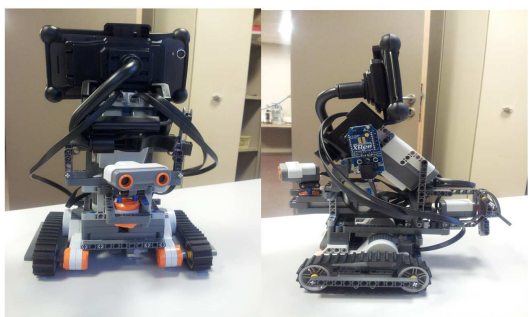

Fig. 6. Lego Mindstorms NXT robot

The robot sends information and receives commands through an XBee module and the obstacle detection is performed by the ultrasonic sensor. When the robot perceives an obstacle less than $40 \mathrm{~cm}$ away, it notifies the human. But when the distance is less than $25 \mathrm{~cm}$, it has the authority to avoid the obstacle automatically and no longer takes into account the human's commands. If the distance becomes less than $10 \mathrm{~cm}$, the robot stops. When the user sends a command the robot performs a $45^{\circ}$ turn. When the robot avoids an obstacle it performs a $20^{\circ}$ turn. We assume that it is not a problem for the robot to repeat $20^{\circ}$ turn to have a more adjusted trajectory, but it is difficult for the user to repeatedly issue turning commands via the BCI without becoming overloaded.

\section{2) Control interface}

Figure 7 presents the visual interface used by the user in order to have a video feedback provided by the robot about the environment, and the emulated haptic feedback to send the command (right or left) to the robot.

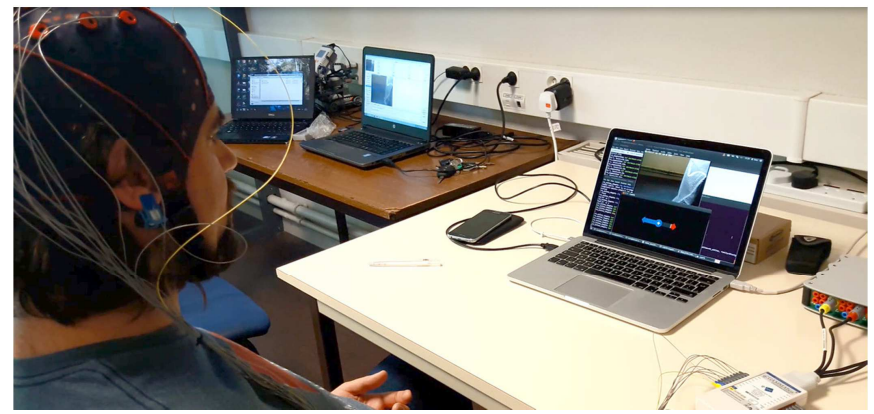

Fig. 7. BCI/Emulated haptic control interface. Visual feedback is provided about the state of the BCI system as well as the environment in which the robot is operating.
The user relaxes his hands loosely on his lap and only has to imagine the kinaesthetic movement of his left or the right hand. A grey rectangular "bar" is rendered on the screen and it moves in the direction corresponding to the imagined hand movement. The bar's motion represents the intention of the user if the model used by the BCI is well-trained and is correctly calibrated to the user. The use of such a device asks the user to be very focused on a single task, whilst attempting to avoid contracting any other muscles (including the position of the eyes) in order to avoid disturbance in the signal detection and analysis. For people with tetraplegia, the chance of such accidental disturbance is lower, since they tend not to have volitional control of large muscle groups.

\section{Experimental protocol}

This first experiment has been conducted to verify the ability to use the emulated haptic feedback to improve user-BCI-robot cooperation. Only one user participates in this experiment. He was a well-trained user, who knew the capabilities (and limitations) of both the BCI and the robot.

Four experimental conditions have been defined combining the possibility of shared control with the possibility of emulated haptic feedback.

Table 1. Experimental conditions: each part of the array presents for each agent (Human and robot) their KH (respectively violet and blue rectangle) and their KHC (light grey rectangles). If the agent has the ability to perform a subfunction, " 1 " is written in the rectangle. The common work space focuses here on the visual feedback of the BCI, but the other part of this workspace comprises a live video stream of the environment from the robot's perspective.

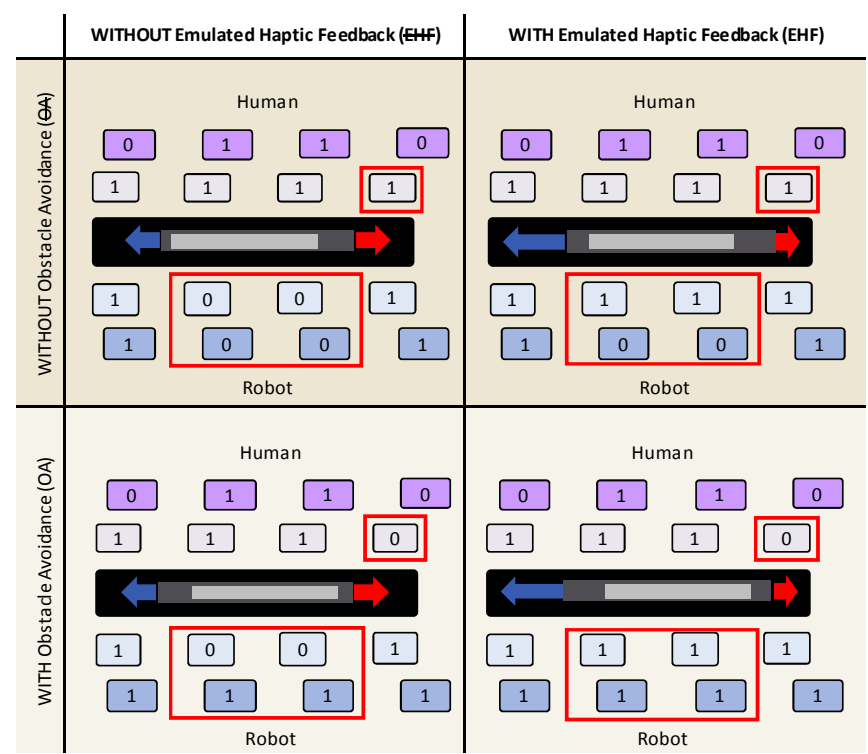

The four conditions are extracted using the cooperative system model (cf. Table 1/Fig. 3). The Table 1 underlines the increase of the level of automation on the $\mathrm{KH}$ (vertical increase) and on the KHC (horizontal increase) [10].

Two consecutive attempts were performed for each experimental condition (conditions were randomized). The results are presented in the next part. 


\section{RESULTS AND DISCUSSION}

The results describe both the objective and subjective data recorded during the experiments. First, the main result concerns the inability of the user to complete the experimental condition with the lower levels of automation ( $\Theta A$ and EHF of Table 1). Several attempts were made, but it was simply too difficult to directly teleoperate the robot without any assistance. Therefore, all results focus on three experimental conditions in which the task was actually completed: OAEHF, OA-EHF, OA-EHF.

\section{1) Results}

\section{a) Objective data}

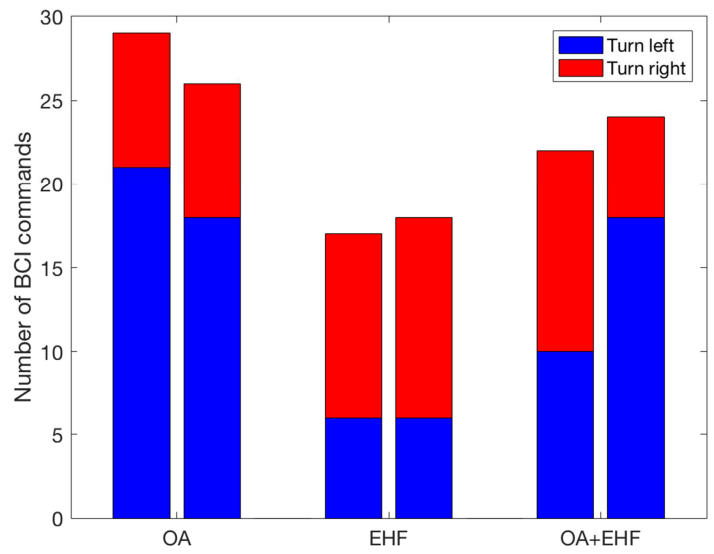

Fig. 8. Number of BCI commands according to the three experimental conditions. Left bar in each pair is the first attempt, right bar is the second attempt

Whilst it was not possible to complete the task in teleoperation, adding obstacle avoidance (OA) made it possible. When using emulated haptic feedback (EHF) instead of OA the performance was even better. However combining the two $(\mathrm{OA}+\mathrm{EHF})$ did not yield an improved performance.

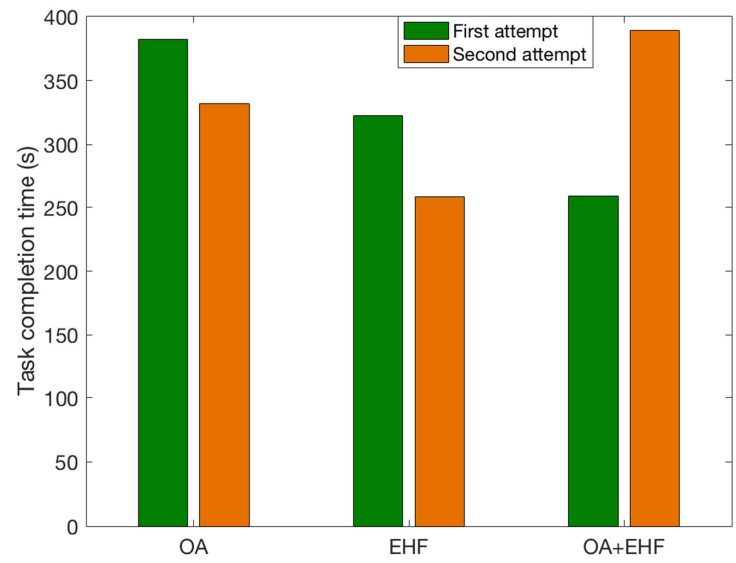

Fig. 9. Task completion time according to the three experimental conditions.

The task completion time followed a similar, though less pronounced, trend to the number of commands. Again, emulated haptic feedback yielded the fastest overall task performance.

\section{b) Results stemming from subjective data records}

\section{Questionnaires}

The questionnaire has been built using the cooperative agent model. Questions relate to how the user perceives the robot's KH, how the user perceives the robot/BCI's KHC, how the robot/BCI perceives the user's $\mathrm{KH}$ and how the robot/BCI perceives the user's KHC, each time on the four sub-functions. The participant had to answer by selecting a value between 1 and 7 (cf. Fig. 10).

\begin{tabular}{llllllllll|}
\hline "To what extent do you think the robot is able to perceive the situation?" \\
Not at all & 1 & 2 & 3 & 4 & 5 & 6 & 7 & Absolutely
\end{tabular}

Fig. 10. Example of a question related to the robot's KH about information gathering.

Questionnaire results underline that the remote control experimental condition score is always higher than other experimental conditions (Average: OA-EHF: 5.07; OA-EHF: 4.27; OA-EHF: 3.93). The questions about the perception by the user of the Robot/BCI KHC highlight the greater differences between conditions (Average of standard deviation by type of question: Robot's KH evaluated by user: 0.67 ; Robot's KHC evaluated by user: 1.4; User's KH evaluated by Robot: 0.0 ; User's KHC evaluated by Robot: 0.8 ). In the non emulated haptic feedback condition (EHF), answers underline the inability for the user to have information about his cooperation with the robot; it is only possible with action but it is not sufficient and often too late to adapt his behavior (Average: OA-EHF: 5.75; OA-EHF: 3.0; OA-EHF: 4.25). Finally, the feeling of "being in control" is better in the remote control condition than in the other conditions (OA-EHF: 6.0; OA-EHF: 3.0; OA-EHF: 3.0).

\section{Video records}

We have also analyzed a combination of objective data (logs of the robot's obstacle detection and decision making; user's decision making), and subjective data (video with the user behavior; visual feedback; and verbal comments).

The videos and comments highlight very interesting situations. The first one deals with the nice cooperation when the user sends a command $\left(45^{\circ}\right.$ right or left) to adjust the trajectory to reach the target, and when the robot further adjusts this trajectory $\left(20^{\circ}\right.$ right or left $)$ to avoid obstacle. The second one deals with the robot's obstacle avoidance when the user sends a "bad" command (that would result in a collision), especially without emulated haptic feedback. The shared control condition always allowed the robot to avoid stopping when the obstacle is too close, and instead corrects the user's mistake. Nevertheless, the robot does not know the final target, and this lack of explicit knowledge means that it can select the wrong direction when it avoids an obstacle. In this case, the user has to correct the robot, by asking it to pass on the opposite side by sending several corrective commands. This increased workload and frustration. However, under the EHF mode, the user's mistake could be directly caused by an instantaneous update of the arrow lengths on the visual feedback (the length depends on the distance to the obstacle). When the grey rectangle of the EHF is at the certain position and the arrows length change, the rectangle could be very 
close to an arrow and the user may not have sufficient time to react, causing a command to be sent even if the user did not want it.

\section{2) Discussion}

The user was unable to complete the task using pure teleoperation, i.e. without shared control and/or emulated haptic feedback. Consequently, the best performance was attained using emulated haptic feedback, but without shared control. Indeed, in the shared control condition the robot proactively avoids obstacles in the direction opposite to the obstacle position; but this direction could appear to be unsuitable for the final goal, which is only explicitly known by the user. So the user had to send several corrective commands to modify the direction (e.g. 180 degrees to go back and pass the obstacle on the other side). The robot is only able to avoid obstacles according to its current trajectory, because it is unaware of potential future commands from the user. A further complicating matter is that the BCI may send a command that the user did not intend to send.

The emulated haptic feedback is driven by the same sensor data as the shared control, however rather than taking authority away from the user and automatically avoiding obstacles, it modulates the effort required to deliver a command. In this way, the user retains the final control authority and is able to either follow the robot current direction or actively ignore it.

By proving the user with the robot's perceived distance to an obstacle, the human can infer the robot's "intention" to avoid the obstacle or not. However, the user does not know when or exactly how this will occur, especially if the obstacle is directly in front of the robot. Nevertheless, it is complicated for the user to have such additional information on the display, because the user should watch information by moving eyes, which could in turn degrade the EEG signal quality.

\section{CONCLUSIONS AND PERSPECTIVES}

These experiments underline the abilities to emulate the haptic concept to control a robot with a brain computer interface. However, the validation has been more technical than regarding acceptance and performance. More participants are required to evaluate the combined $\mathrm{BCI} /$ robot system. Nevertheless, positive and negative points have already been underlined. The remote control condition combined with the emulated haptic feedback initially seems to be the best condition. But it is perhaps due to a lack in the robot's $\mathrm{KH}$ about the tactical level, or a lack in robot's KHC in its interaction with human. Some improvements will be implemented in the behavior of the robot in order to compensate the selection of "wrong" directions regarding target position. An intermediate level of activity could be found between the tactical and operational one to identify the main direction the user intends to follow.

Another perspective could be the update of the level of automation of the Robot/BCI system, regarding the difficulty of task (number of obstacles) and the fatigue of the user. It could be represented by the size of the grey rectangular bar: the larger the bar, the lower the level of automation and the authority of the robot. Nevertheless, the update of the level could be proposed by the system and validated by the user but this would require an additional BCI command.

\section{REFERENCES}

[1] M. P. Pacaux-Lemoine, "Human-Machine Cooperation Principles to Support Life-Critical Systems Management," in P. Millot (ed) Risk Management in Life-Critical Systems, ISTE-Wiley London, pp. 255277, 2014.

[2] J.-M. Hoc and M.-P. Lemoine, "Cognitive Evaluation of Human-Human and Human-Machine Cooperation Modes in Air Traffic Control,” Int. J. Aviat. Psychol., vol. 8, no. 1, pp. 1-32, 1998.

[3] M. P. Pacaux, S. Debernard, A. Godin, B. Rajaonah, F. Anceaux, and F. Vanderhaegen, "Levels of automation and human-machine cooperation: Application to human-robot interaction," IFAC Proc. Vol., vol. 18, no. PART 1, pp. 6484-6492, 2011.

[4] R. Parasuraman, T. B. Sheridan, and C. D. Wickens, "A Model for Types and Levels of Human Interaction with Automation," vol. 30, no. 3, pp. 286-297, 2000.

[5] L. Habib, M.-P. Pacaux-Lemoine, and P. Millot, "Towards adaptability of levels of automation with Human-Machine cooperation approach," in IEEE SMC, pp. 1081-1086, 2016.

[6] J. Rasmussen, "Skills, Rules, and Knowledge; signals, Signs, and Symbols, and Other Distinctions in Human Performance Models," IEEE Trans. Syst. Man. Cybern., vol. 13, no. 3, pp. 257-266, 1983.

[7] M.P. Pacaux-Lemoine and S. Debernard. "A Common Work Space to support the Air Traffic Control”.Control Engineering Practice, a Journal of IFAC, 10, 571-576, 2000.

[8] P. Millot and M. P. Pacaux-Lemoine, "A common work space for a mutual enrichment of human-machine cooperation and team-situation awareness," Proceedings of IFAC HMS 2013, vol. 12, no. PART 1, pp. 387-394, 2013.

[9] M.-P. Pacaux-Lemoine and M. Itoh, "Towards vertical and horizontal extension of shared control concept," IEEE International Conference on Systems, Man, and Cybernetics, Hong Kong, China, 2015.

[10] L. Habib, M.P. Pacaux-Lemoine and P. Millot. "A method for designing levels of automation based on a human-machine cooperation model". In IFAC World Congress. Toulouse, France, 2017.

[11] Flemisch F.O., Adams C.A., Conway S.R., Goodrich K.H., Palmer M.T., Schutte M.C. (2003) The H-Metaphor as a Guideline for Vehicle Automation and Interaction. Report No. NASA/TM-2003-212672. NASA Langley.

[12] Pacaux-lemoine M., Flemisch F. (2016). Layers of Shared and Cooperative Control, assistance and automation. IFAC Analysis, Design and Evaluation of Human-Machine Systems, Kyoto, Japan, august.

[13] Leeb, R., Tonin, L., Rohm, M., Desideri, L., Carlson, T., Millán, J.D.R. (2015). Towards independence: A BCI telepresence robot for people with severe motor disabilities. Proceedings of the IEEE, 103 (6), 969982.

[14] Carlson, T., Millán, J.D.R. (2013). Brain-Controlled Wheelchairs: A Robotic Architecture. IEEE Robotics and Automation Magazine, 20 (1), $65-73$.

[15] Lee, K., Liu, D. , Perroud, L., Chavarriaga R. and Millán, J.d.R. (2017) A Brain-Controlled Exoskeleton with Cascaded Event-Related Desynchronization Classifiers, in Robotics and Autonomous Systems, vol. 90, p. 15-23.

[16] J. d. R. Millán, F. Renkens, J. Mourinño, and W. Gerstner, "Noninvasive brain-actuated control of a mobile robot by human EEG," IEEE Trans Biomed Eng, vol. 51, no. 6, pp. 1026-1033, 2004.

[17] F.Galán, P.W.Ferrez, F.Oliva, J.Guàrdia, and J.d.R.Millán, "Feature extraction for multi-class BCI using canonical variates analysis," in IEEE Int Symp Intelligent Signal Processing, 2007.

[18] S. Perdikis, H. Bayati, R. Leeb, and J. d. R. Millan, "Evidence accumulation in asynchronous BCI," International Journal of Bioelectromagnetism, vol. 13, no. 3, pp. 131-132, 2011.

[19] Abbink, D. A., Mulder, M., \& Boer, E. R. (2012). Haptic shared control: smoothly shifting control authority?. Cognition, Technology \& Work, 14(1), 19-28. 